\title{
Estudo do endotélio corneano em cirurgias de cataratas duras: extração extracapsular planejada da catarata e facoemulsificação
}

\author{
Endothelial damage after planned extracapsularcataract extraction \\ and phacoemulsification of hard cataracts
}

Simone Stump ${ }^{1}$

Walton Nosé ${ }^{2}$

Trabalho realizado na Universidade Federal de São Paulo - UNIFESP. Departamento de Oftalmologia. Setor de Catarata.

${ }^{1}$ Pós-graduanda, nível doutorado, Departamento de Oftalmologia. Universidade Federal de São Paulo - UNIFESP - São Paulo (SP) - Brasil.

${ }^{2}$ Livre docente do Departamento de Oftalmologia da UNIFESP - São Paulo (SP) - Brasil. Professor titular do Departamento de Oftalmologia. Universidade Metropolitana de Santos - UNIMES - Santos (SP) - Brasil. Diretor da Eye Clinic Day Hospital.

Endereço para correspondência: Simone Stumpf. Rua Vicente da Fontoura, 3007/405 - Porto Alegre (RS) CEP 90640-003

E-mail: sistumpf@terra.com.br

Recebido para publicação em 02.08.2005

Versão revisada recebida em 15.12.2005

Aprovação em 10.01.2006

\begin{tabular}{|l|}
\hline RESUMO \\
\hline Objetivo: Avaliar o dano endotelial em cirurgias de catarata com núcleo \\
duro, comparando as técnicas da extração extracapsular planejada da \\
catarata e a facoemulsificação. Métodos: Estudo prospectivo, incluindo \\
41 pacientes com catarata senil e núcleo muito duro, que foram divididos \\
aleatoriamente em dois grupos: grupo 1 (21 pacientes) foi submetido à \\
extração extracapsular da catarata (EECC) e grupo 2 (20 pacientes) à \\
facoemulsificação (FACO); em todos casos foram implantadas lentes \\
intra-oculares no saco capsular. Exame oftalmológico completo, micros- \\
copia especular e paquimetria foram realizados no pré-operatório e com \\
um, três e seis meses de pós-operatório. Perda endotelial, pleomorfismo, \\
polimegatismo e paquimetria foram aspectos estudados. Resultados: \\
Nos dois grupos, ocorreu perda endotelial significativa, comparando os \\
valores pré e pós-operatórios, mas não houve diferença entre os tempos \\
pós-operatórios (um, três e seis meses). Não houve diferença estatística \\
da perda endotelial entre os grupos da extração extracapsular da catarata \\
e facoemulsificação, em todos tempos estudados. A média de perda \\
endotelial com seis meses de cirurgia no grupo 1 (EECC) foi de 28,50\% \\
e no grupo 2 (FACO), de 34,77\%. Não houve diferença significativa nas \\
medidas da paquimetria, polimegatismo e pleomorfismo, entre os dois \\
grupos. Conclusões: As diferenças percentuais da densidade endotelial, \\
polimegatismo, pleomorfismo paquimetria não foram estatisticamente \\
significantes entre o grupo da extração extracapsular da catarata e da \\
facoemulsificação, em todos tempos estudados. \\
\hline
\end{tabular}

Descritores: Endotélio da córnea; Microscopia; Extração da catarata Facoemulsificação; Terapia por ultra-som

\section{INTRODUÇÃO}

A indicação da melhor técnica para tratamento das cataratas senis, com núcleos duros, permanece em debate ${ }^{(1-3)}$. De um lado, a facoemulsificação, com a vantagem de precisar de menor incisão e apresentar uma recuperação mais rápida ${ }^{(4)}$ e a desvantagem de necessitar, para emulsificação dos núcleos duros, de maior quantidade de energia ultra-sônica, o que está correlacionado com aumento da lesão ao endotélio corneano ${ }^{(5)}$. De outra parte, a extração extracapsular, necessitando de incisões maiores tem uma recuperação mais lenta, todavia não utiliza ultra-som e, tecnicamente exige menor manipulação intra-ocular ${ }^{(1)}$. 
A descompensação da córnea, após cirurgia de catarata, continua sendo indicação de transplante penetrante de córnea, sobretudo nos casos de maior trauma cirúrgico e consequiente dano endotelial ${ }^{(6)}$.

O endotélio corneano é uma camada celular única, com aproximadamente 500 mil células, localizado na superfície posterior da córnea. Ele deriva da crista neural e tem baixa capacidade de regeneração. Havendo perda celular, as células sobreviventes preenchem as lacunas resultantes, aumentando de tamanho, perdendo sua regularidade em tamanho (polimegatismo) e forma (pleomorfismo $)^{(7)}$.

O estudo da morfologia e densidade endotelial pode ser realizado através da microscopia especular da córnea. As alterações endoteliais são consideradas importante parâmetro para avaliar trauma e para estimar a segurança de uma técnica cirúrgica $^{(7)}$. A análise da forma e regularidade do endotélio é um sensível indicador do dano endotelial, sobressaindo-se a contagem da densidade endotelial isolada ${ }^{(8)}$.

O pobre funcionamento do endotélio corneano leva ao edema de córnea e conseqüente aumento progressivo da espessura corneana. A paquimetria avalia indiretamente o funcionamento do endotélio corneano, entretanto, mais próximo à realidade que somente a avaliação da anatomia celular, pois a espessura corneana está diretamente relacionada ao funcionamento das células endoteliais ${ }^{(7)}$.

\section{OBJETIVO}

Avaliar e comparar prospectivamente as alterações do endotélio da córnea em cirurgias de catarata com núcleo muito duro, com as técnicas cirúrgicas de extração extracapsular planejada e facoemulsificação.

\section{MÉTODOS}

Estudo prospectivo realizado em 41 olhos de 39 pacientes, sendo 12 homens e 27 mulheres com idades entre 54 e 88 anos (média de 75 anos). Os pacientes foram divididos aleatoriamente, através de sorteio, em dois grupos. No grupo $1(\mathrm{n}=21)$, a técnica empregada foi a extração extracapsular da catarata (EECC) e no grupo 2 ( $n=20)$, a técnica utilizada foi a facoemulsificação (FACO). Todas as cirurgias foram realizadas por um dos autores (WN).

Os pacientes foram selecionados do ambulatório de catarata do Departamento de Oftalmologia da Escola Paulista de Medicina da Universidade Federal de São Paulo (EPM-UNIFESP). Este estudo foi avaliado e aprovado pelo Comitê de Ética da Universidade Federal de São Paulo. As cirurgias foram realizadas nos anos de 2000 e 2001.

Os critérios de inclusão foram: presença de catarata senil, total, que, ao exame à lâmpada de fenda, apresentavam núcleo do cristalino com coloração marrom escura; possibilidade de retorno aos exames por pelo menos seis meses.
Os critérios de exclusão foram: presença de patologia ocular concomitante com a catarata; cirurgia ocular prévia no olho estudado; portadores de diabete melito; casos que tiveram complicações per ou pós-operatória; pacientes que não fizeram seguimento pós-operatório de seis meses.

No pré-operatório, os pacientes foram submetidos a exame oftalmológico completo e exames complementares: ultra-som ocular (Alcon), biometria (Humphrey), microscopia especular de contato (Bio-Optics LSM-2100C), paquimetria ultra-sônica (Humphrey) e documentação fotográfica do cristalino (Topcon).

A dilatação pupilar foi realizada 30 minutos antes da cirurgia, com colírio de tropicamida (Mydriacyl ${ }^{\circledR} 1 \%$ ) e colírio de cloridrato de fenilefrina (Fenilefrina ${ }^{\circledR} 10 \%$ ). Utilizou-se baroftalmo, durante 15 minutos prévios ao procedimento.

Empregou-se a anestesia tópica para os dois grupos. Associou-se a sedação sugerida por alguns autores ${ }^{(9)}$, empregando midazolam via sublingual.

A técnica "stop and chop"(10) foi empregada nas cirurgias de facoemulsificação.

O aparelho de facoemulsificação utilizado foi o Legacy ${ }^{\circledR}$ 20000 (Alcon). Usou-se o Max Vac ${ }^{\circledR}$ Cassette. A ponteira do facoemulsificador foi a MicroTip ${ }^{\circledR}$ ABS $^{\mathrm{TM}}$, modelo Kelman, com inclinação de $30^{\circ}$.

Foi padronizado para todas as cirurgias o uso de: viscoelásticos: Hidroxipropilmetilcelulose 2\% - (Metilcelulose $^{\circledR} 2 \%$ Ophthalmos) e Hialuronato de sódio $1 \%$ - (Provisc ${ }^{\circledR}$ - Alcon); Solução salina balanceada (BSS) - BSS ${ }^{\circledR}$ (Alcon); lente intraocular de câmara posterior (Vision Tech, modelo 15HOB); não utilização intra-ocular de corantes, midriáticos ou mióticos.

Os pacientes receberam colírio de diclofenaco sódico (Still ${ }^{\circledR}$ - Allergan) e colírio de tobramicina e dexametasona (Tobradex ${ }^{\circledR}$ - Alcon), para o pós-operatório.

Os retornos pós-operatórios foram agendados com 1, 7, 30, 90 e 180 dias, sendo os exames oftalmológicos realizados pelo pesquisador principal. Nos retornos de 30, 90 e 180 dias, também foram feitos exames de microscopia especular de contato e paquimetria.

Foram capturadas três imagens endoteliais, com boa nitidez da área corneana central dos pacientes integrantes do estudo, em cada tempo proposto. A análise das imagens foi feita com o programa Thumper do microscópio especular BioOptics ${ }^{\circledR}$, avaliando a densidade endotelial, o polimegatismo e o pleomorfismo.

Realizou-se a tomada de cinco medidas paquimétricas centrais da córnea para cada olho, em cada tempo proposto. Utilizou-se a média aritmética das medidas.

A acuidade visual foi medida com a melhor correção possível, utilizando-se a escala de Snellen. Fez-se a conversão da acuidade visual medida para o equivalente $\log$ MAR, para a obtenção da média da acuidade visual dos grupos e realização dos cálculos estatísticos ${ }^{(11)}$.

Outros dados, como tempo de cirurgia e volume de solução salina balanceada (BSS), foram controlados. No grupo submetido à facoemulsificação, também registrou-se o tempo e o porcentual de ultra-som utilizados em cada cirurgia. 
Foram estudadas possíveis correlações entre algumas variáveis de cada grupo e a perda endotelial com seis meses de cirurgia. No grupo da EECC, foram correlacionadas as variáveis: idade do paciente, volume de BSS e perda endotelial com seis meses de cirurgia. No grupo da FACO: idade do paciente, volume de BSS, tempo de ultra-som, percentual de ultra-som e perda endotelial com seis meses de cirurgia.

\section{MÉTODO ESTATÍSTICO}

Para avaliar possíveis diferenças entre os tempos, tanto no grupo FACO como no grupo EECC, para cada variável previamente definida, utilizou-se o teste não paramétrico para " $\mathrm{K}$ " amostras não independentes, de Friedman. Este foi complementado, quando necessário, pelo teste de comparações múltiplas.

Para comparar as diferenças entre os grupos para cada variável nos tempos estudados, usou-se o teste para duas amostras independentes de Mann-Whitney.

Para estudar possíveis correlações entre as variáveis previamente definidas, empregou-se o teste não paramétrico de correlação por postos, de Spearman.

O nível de significância para a rejeição da hipótese de nulidade foi fixado sempre num valor menor ou igual do que $0,05(5 \%)$.

Quando a estatística calculada apresentou significância, usou-se um asterisco (*) para caracterizá-la; caso contrário, isto é, quando se mostrou não significante, usou-se N.S. As médias foram calculadas e apresentadas a título de informação.

\section{RESULTADOS}

Não houve diferença estatisticamente significante, na comparação dos grupos, quanto à perda endotelial, com um, três ou seis meses de seguimento pós-operatório. Com seis meses de pós-operatório a perda endotelial observada no grupo da EECC foi de 28,50\% e no grupo da FACO de $34,77 \%$ $(\mathrm{p}=0,361)$. No gráfico 1 observa-se a variação da densidade endotelial observada nos dois grupos, nos tempos pré e pósoperatórios.

Os índices de polimegatismo e pleomorfismo não tiveram variação significativa na análise comparativa entre os grupos e em cada grupo, nos tempos estudados.

No grupo da EECC observou-se um aumento significativo nas medidas paquimétricas pós-operatório em relação ao préoperatório ( $\mathrm{p}=0,033)$. Já, no grupo da FACO, não houve variação significativa nas medidas de espessura corneana, nos tempos propostos. Também não se encontrou diferença significante quanto à análise das paquimetrias entre os grupos ( $\mathrm{p}=0,354)$ (Gráfico 2).

A média da acuidade visual corrigida com um e três meses de cirurgia foi melhor no grupo da facoemulsificação; mas com seis meses, não houve diferença significativa entre o grupo da FACO e da EECC. Com seis meses de pós-operatório, a média de acuidade visual corrigida foi de $0,87(\log M A R=0,07)$ nas cirurgias de FACO e de 0,81 $(\log$ MAR=0,10) nas EECC $(\mathrm{p}=0,35)($ Gráfico 3$)$.

O tempo médio de cirurgia foi 19,7 min, no grupo 1 e 15,6 min, no grupo $2(\mathrm{p}=0,008)$. A média do volume de solução salina balanceada, utilizada nas cirurgias, foi $59,3 \mathrm{ml}$, no grupo da EECC, e 139,5 ml, na FACO (p<0,001). Estas diferenças são estatisticamente significantes. A média de tempo de ultra-som nas cirurgias de facoemulsificação foi 3,10 $\pm 1,03 \mathrm{~min}$; sendo

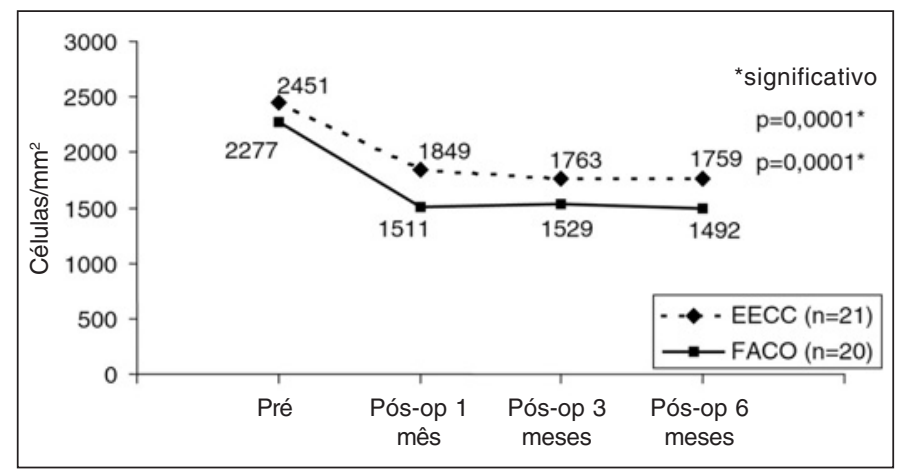

Gráfico 1 - Média da densidade endotelial dos grupos submetidos à EECC e à FACO, nos tempos pré e pós-operatórios

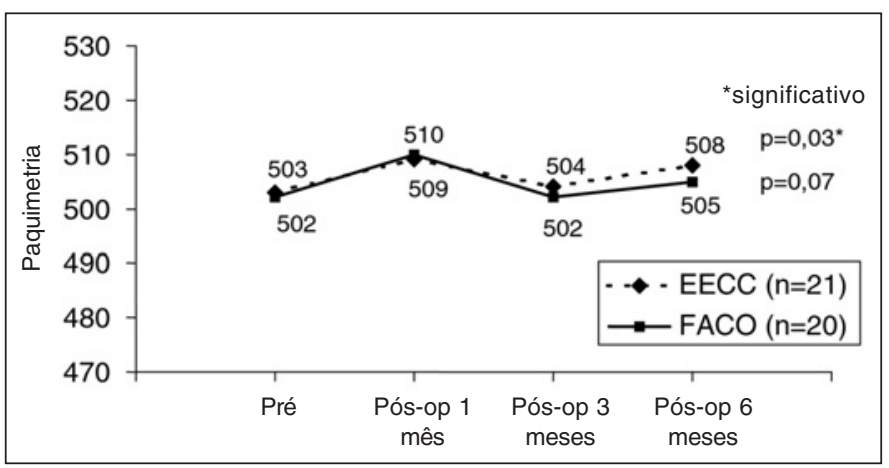

Gráfico 2 - Média das medidas paquimétricas ( $\mu \mathrm{m})$ dos grupos submetidos à EECC e à $\mathrm{FACO}$, nos tempos pré e pós-operatórios

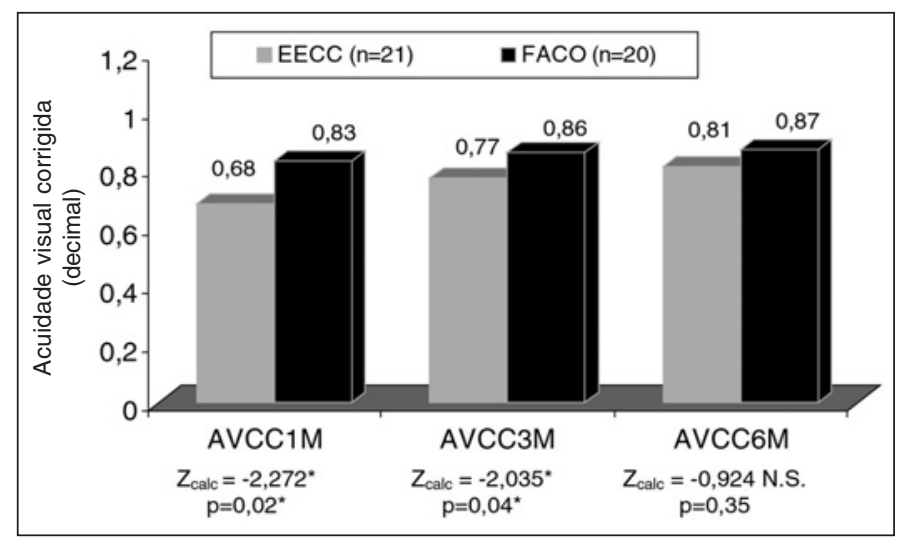

Gráfico 3 - Média da acuidade visual com correção do grupo da EECC e da FACO, nos tempos pós-operatório 
que a média do porcentual de ultra-som (AP-“average power") foi $36,0 \pm 4,8 \%$.

No grupo da EECC, não se encontrou correlação da perda endotelial com seis meses de pós-operatório, com a idade dos pacientes e o volume de BSS utilizado nas cirurgias. No grupo da FACO, houve correlação positiva significante da perda endotelial e o tempo de ultra-som ( $\mathrm{p}=0,049)$, mas não foi significativa em relação à idade dos pacientes, volume de BSS e porcentual de ultra-som empregados nas cirurgias.

\section{DISCUSS ÃO}

Nos primórdios da facoemulsificação, sem a proteção endotelial dos agentes viscoelásticos e o emprego do ultrasom em câmara anterior, a perda endotelial era maior que na extração extracapsular da catarata ${ }^{(12-13)}$.

Com a modernização da facoemulsificação, a melhora tecnológica dos aparelhos, o emprego de agentes viscoelásticos, o surgimento de técnicas de fratura do núcleo dentro do saco capsular e o uso do ultra-som na câmara posterior, a perda endotelial baixou consideravelmente $(7-12 \%)^{(14-17)}$.

Estudos que analisaram prospectivamente o dano endotelial ocorrido em cirurgias de cataratas não densas, comparando a EECC e a facoemulsificação, observaram perda endotelial similar com as técnicas $(7-12 \% \text { e } 7-11 \% \text {, respectivamente) })^{(14-16)}$. Esses trabalhos, assim como outros, não classificam as cataratas e a dureza dos núcleos lenticulares, limitando a análise comparativa com este estudo.

A facoemulsificação em catarata muito dura apresenta várias dificuldades: baixa visibilidade, que dificulta a realização da capsulorrexe; cápsula anterior frágil, que é lesada com facilidade; núcleo de tamanho grande, diminuindo o espaço para manipulação dos instrumentos e tensionando o saco capsular; fibras lenticulares muito coesivas e elásticas, dificultando a quebra do núcleo e aumentando o risco de fragmentos nucleares tocarem o endotélio; e necessidade de tempo prolongado de ultra-som, aumentando o risco de lesão endotelial e de queimadura corneana ${ }^{(18)}$.

A perda endotelial encontrada no grupo da facoemulsificação foi maior que a observada em outros estudos que também avaliaram esta técnica em núcleos duros ${ }^{(2-3)}$. Alguns autores estudaram prospectivamente o efeito da facoemulsificação em 167 casos de cataratas densas. Obtiveram uma perda endotelial, num ano de pós-operatório, de 13,05\% nas cataratas duras e $15 \%$ nas cataratas negras. A técnica utilizada foi a "step-bystep chop in situ and separation", desenvolvida pelos autores para manejar os núcleos duros. Utilizaram nas suas cirurgias o aparelho de facoemulsificação Legacy ${ }^{\circledR} 20000$, viscoelástico hidroxipropilmetilcelulose $2 \%$ (Viscomet $^{\circledR}$ ) e solução salina balanceada $\left(\mathrm{BSS}^{\circledast}\right)^{(3)}$. Outro trabalho analisou uma série de 15 olhos com catarata negra, encontrando uma média de perda endotelial de $25,59 \%$. Houve grande variação na percentagem de perda, desde pacientes que não diminuíram sua densidade celular até casos com $48,52 \%$ de perda com três meses de cirurgia. A técnica utilizada foi a "stop and chop", o aparelho de facoemulsificação foi o Legacy ${ }^{\circledR} 20000$ e o viscoelástico utilizado foi o hialuronato de sódio 3,0\% - sulfato de condroitina $4,0 \%\left(\text { Viscoat }^{\circledR}\right)^{(2)}$.

Em um estudo multicêntrico, comparativo, entre a facoemulsificação $(n=223)$ e a extração extracapsular $(n=210)$, não se encontrou diferença significativa quanto à perda endotelial entre as duas técnicas (10,5 e 9,1\%, respectivamente). $\mathrm{Na}$ analise de um subgrupo, 45 casos, com catarata dura; observou-se maior perda endotelial, nos núcleos duros, com a técnica de facoemulsificação $[18,9 \%(n=19)$ contra $11,8 \%(n=26)]$. Quando se estudou somente os casos com perda endotelial superior a $15 \%$, houve 6 casos de extração extracapsular com média de perda de $23,1 \%$ e 10 casos de facoemulsificação, com média de perda de $52,6 \%{ }^{(19)}$.

Apesar da dureza do núcleo lenticular não ser um fator considerado nos trabalhos que avaliam a densidade endotelial em cirurgias de extração extracapsular da catarata ${ }^{(16,20)}$, o tamanho dos núcleos duros representa uma adversidade na hora de sua extração, aumentando a manipulação e o risco de toque do núcleo ao endotélio e conseqüente lesão a esta camada ${ }^{(5)}$. Outro fator que deve ser considerado é o local da abordagem cirúrgica, a incisão utilizada foi limbar, que tende a perder mais células que a abertura escleral ${ }^{(21)}$.

Embora o mesmo cirurgião, com experiência em ambos procedimentos, tenha realizado todas as cirurgias, reduzindo a variabilidade inerente à habilidade cirúrgica, as dificuldades técnicas da facoemulsificação em núcleos duros são maiores que a extração do núcleo inteiro.

Não houve variação significativa dos índices de polimegatismo e pleomorfismo pré e pós-operatórios nos dois grupos estudados. Isto está de acordo com o observado por outros autores, que também não observaram variação dos índices morfométricos após cirurgias de cataratas duras ${ }^{(3,19)}$.

Nesta pesquisa, encontrou-se variação significativa nos valores paquimétricos no grupo da EECC. O teste de Comparações Múltiplas não conseguiu detectar as diferenças, mas é sugestivo que a diferença seja da média paquimétrica préoperatória $(503 \mu \mathrm{m})$, em comparação à média do primeiro mês pós-operatório $(509 \mu \mathrm{m})$. Este aumento transitório da espessura corneana demonstrou que a função endotelial não estava totalmente restabelecida com um mês de pós-operatório no grupo da EECC. As medidas paquimétricas retornaram aos valores pré-operatórios com três meses da cirurgia, indicando uma recuperação da função de bomba endotelial.

Apesar do grupo da EECC ter apresentado perda endotelial equiparável ao grupo da FACO, as medidas paquimétricas mantiveram-se alteradas por mais tempo, demonstrando uma recuperação da função endotelial mais lenta neste grupo.

Ocorreu uma recuperação visual mais rápida no grupo submetido à facoemulsificação. Nos primeiros períodos de seguimento (um e três meses), observou-se melhor acuidade visual corrigida no grupo submetido à facoemulsificação. Esta diferença desapareceu no sexto mês, quando os grupos obti- 
veram resultados semelhantes nesta variável. Neste aspecto, a facoemulsificação apresenta vantagens em relação à extração extracapsular convencional, por necessitar de menor incisão a indução de astigmatismo pós-operatório é menor ${ }^{(4,22)}$.

A correlação positiva entre a perda endotelial e o tempo de ultra-som, utilizado nas cirurgias de facoemulsificação deste estudo, confirma os achados de outras pesquisas que apontam a energia ultra-sônica como um fator lesivo ao endotélio corneano $^{(5,17)}$.

Em um estudo prospectivo, com objetivo de identificar os principais fatores de risco para dano endotelial durante a facoemulsificação, avaliou-se a densidade endotelial no préoperatório e com três meses de cirurgia, em 843 olhos. Observou-se correlação da perda endotelial com idade avançada, diâmetro pupilar pequeno, núcleo lenticular duro, núcleo grande, grande volume de irrigação e tempo prolongado de ultra-som. A dureza do núcleo do cristalino foi identificada como o principal risco para perda endotelial ${ }^{(5)}$.

Neste estudo, foi empregada uma ponteira fina, com $0,9 \mathrm{~mm}$ de diâmetro, que ajuda na prevenção da queimadura da córnea $^{(23)}$, contudo dificulta na aspiração dos fragmentos duros ${ }^{(24)}$. Hoje, com a disponibilidade do uso de ponteira padrão (diâmetro de $1,1 \mathrm{~mm}$ ) associada à luva de alta infusão, haveria maior eficiência na aspiração e na redução no tempo de ultra-som.

\section{CONCLUSÕES}

A cirurgia de cataratas duras continua representando uma adversidade ao cirurgião. A experiência do cirurgião é relevante na escolha da técnica cirúrgica a ser empregada. Importa considerar que as duas técnicas apresentam resultados parecidos, a médio prazo, e que a facoemulsificação é tecnicamente mais difícil. Urge que se indique a cirurgia mais precocemente.

\section{AGRADECIMENTOS}

Agradecimento à direção, colegas e funcionários da Eye Clinic pela importante colaboração neste estudo. Agradecemos também ao Laboratório Alcon, à Farmácia Ophthalmos e à Vision Tech pelo gentil suprimento de materiais utilizados nas cirurgias desta pesquisa.

\section{ABSTRACT}

Purpose: To evaluate and compare the endothelial damage after planned extracapsular cataract extraction (ECCE) and phacoemulsification of very hard cataracts. Methods: In this prospective, randomized study, 41 patients with age-related and very hard cataract were divided into two groups: in group 1 (21 patients) an extracapsular cataract extraction was performed and in group 2 (20 patients), phacoemulsification. In both groups, intraocular lenses were implanted in the capsular bag.
Preoperatively and 1, 3 and 6 months postoperatively, a complete ophthalmological examination, endothelial specular microscopy, and ultrasonic pachymetry were done. Endothelial cell loss, pleomorphism, polymegathism and corneal thickness were studied. Results: Both groups presented an endothelial cell loss in the postoperative time, as compared with the preoperative values, but there were no significant differences among the postoperative values (1, 3 and 6 months). Six months after surgery, mean cell loss was $28.50 \%$ in group 1 and $34.77 \%$ in group 2 . There were no differences among the indexes of pachymetry, polymegathism and pleomorphism between the two groups. Conclusions: Endothelial response was not statistically different between the two studied groups.

Keywords: Endothelium, corneal; Microscopy; Cataract extraction; Phacoemulsification; Ultrasonic therapy

\section{REFERÊNCIAS}

1. Kimura H, Kuroda S, Mizoguchi N, Terauchi H, Matsumura M, Nagata M. Extracapsular cataract extraction with a sutureless incision for dense cataracts. J Cataract Refract Surg. 1999;25(9):1275-9.

2. Centurion V, Caballero JC, Carrari MJ, Lacava AC. Catarata nigra e facoemulsificação. Rev Bras Oftalmol. 2001;60(10):713-7.

3. Singh R, Vasavada AR, Janaswamy G. Phacoemulsification of brunescent and black cataracts. J Cataract Refract Surg. 2001;27(11):1762-9.

4. Watson A, Sunderraj P. Comparison of small-incision phacoemulsification with standard extracapsular cataract surgery: post-operative astigmatism and visual recovery. Eye. 1992;6(Pt 6):626-9.

5. Hayashi K, Hayashi H, Nakao F, Hayashi F. Risk factors for corneal endothelial injury during phacoemulsification. J Cataract Refract Surg. 1996;22(8):1079-84.

6. Lois N, Kowal VO, Cohen EJ, Rapuano CJ, Gault JA, Raber IM, et al. Indications for penetrating keratoplasty and associated procedures, 1989-1995. Cornea. 1997;16(6):623-9.

7. Waring GO 3rd, Bourne WM, Edelhauser HF, Kenyon KR. The corneal endothelium. Normal and pathologic structure and function. Ophthalmology. 1982;89(6):531-90.

8. Matsuda M, Suda T, Manabe R. Serial alterations in endothelial cell shape and pattern after intraocular surgery. Am J Ophthalmol. 1984;98(3):313-9.

9. Andrade BBA, Forseto AS, Nosé RAM, Kimura A, Nosé W. Uso de sedação sublingual associado à anestesia tópica em facoemulsificação. Rev Bras Oftalmol. 2000;59(4):281-4

10. Koch PS, Katzen LE. Stop and chop phacoemulsification. J Cataract Refract Surg. 1994;20(5):566-70.

11. Holladay JT. Proper method for calculating average visual acuity. J Refract Surg. 1997;13(4):388-91.

12. Graether JM, Davison JA, Harris GW, Watt RH, Widner RR, Sposito V. A comparison of the effects of phacoemulsification and nucleus expression on endothelial cell density. J Am Intraocul Implant Soc. 1983;9(4):420-3.

13. Colin J, Bonnet P. [Comparison of phacoemulsification and manual extracapsular extraction of the lens] Ophtalmologie. 1989;3(3):233-4. French.

14. Ohrloff C, Zubcov AA. Comparison of phacoemulsification and planned extracapsular extraction. Ophthalmologica. 1997;211(1):8-12.

15. Ravalico G, Tognetto D, Palomba MA, Lovisato A, Baccara F. Corneal endothelial function after extracapsular cataract extraction and phacoemulsification. J Cataract Refract Surg. 1997;23(7):1000-5. Comment in: J Cataract Refract Surg. 1997;23(7):967-8.

16. Diaz-Valle D, Benitez del Castillo Sanchez JM, Castillo A, Sayagues O, Moriche M. Endothelial damage with cataract surgery techniques. J Cataract Refract Surg. 1998;24(7):951-5.

17. Walkow T, Anders N, Klebe S. Endothelial cell loss after phacoemulsification: relation to preoperative and intraoperative parameters. J Cataract Refract Surg. 2000;26(5):727-32. 
18. Vasavada AR, Singh R. Surgical techniques for difficult cataracts. Curr Opin Ophthalmol. 1999;10(1):46-52.

19. Bourne RRA, Minassian DC, Dart JKG, Rosen P, Kaushal S, Wingate N. Effect of cataract surgery on the corneal endothelium. Modern phacoemulsification compared with extracapsular cataract surgery. Ophthalmology. 2004;111(4):679-85.

20. Kim JY, Lee JH. The effect of extracapsular cataract extraction using nucleus dislocation into anterior chamber on the corneal endothelium. Korean J Ophthalmol. 1993;7(2):55-8.

21. Freitas LL, Martino DSD, Mori E, Mendonça M, Casanova FHC, Abreu MT Estudo prospectivo comparativo de duas técnicas cirúrgicas de extração extracap- sular planejada de catarata com implante de lente intra-ocular: incisão limbar e incisão escleral tunelizada. Arq Bras Oftalmol. 2001;64(3):239-46.

22. Müller-Jeusen K, Barlinn B, Zimmerman H. Astigmatism reduction: no-stitch $4.0 \mathrm{~mm}$ versus sutured $12.0 \mathrm{~mm}$ clear corneal incision. J Cataract Refract Surg. 1996;22(8):1108-12.

23. Hiroko BM, Shigeto S, Kazuo T. Thermal effect on corneal incisions with different phacoemulsification ultrasonic tips. J Cataract Refract Surg. 1999; 25(1):60-4.

24. Davison JA. Performance comparison of the Alcon Legacy $200001,1 \mathrm{~mm}$ TurboSonics and 0,9 mm MicroTip. J Cataract Refract Surg. 1999;25(10):1382-5.

\title{
42: Reuniäo Anual do Centro de Estudos Offalmologices Gyre de Rezende*
}

\section{2 e 23 de Setembro de 2006}

\section{Centro de Convenções de Ribeirão Preto - SP}

\author{
INFORMAÇÕES: Tels.: (16) 3602-2523 / 3602-2802 com Amélia \\ E-mail: ceoft@hcrp.fmrp.usp.br \\ Home-page: www.fmrp.usp.br/roo
}

*Participaçãa neste evento conta 10 pontos para a revalidaçãa do títula de especialista em oftalmologia 